\title{
ON A SYSTEM OF NONLINEAR PARTIAL DIFFERENTIAL EQUATIONS ARISING IN MATHEMATICAL ECONOMICS
}

\author{
BY MELVYN S. BERGER AND NORMAN G. MEYERS
}

Communicated by L. Nirenberg, April 21, 1966

The theory of utility in the social sciences dates back to studies of Daniel Bernoulli, and can be considered as an attempt to extend the ideas of the calculus of variations into the realm of economics. One approach to utility theory consists of deriving a real-valued utility function from a simple system of non-linear partial differential equations, obtainable empirically from economic data. This point of view has been developed for over a century and has been studied recently for equations with continuously differentiable coefficients by Samuelson [6] and Hurwicz and Uzawa [4]. In this paper we consider the case of "kinked" coefficients i.e. Lipschitz continuous but not necessarily everywhere continuously differentiable (a not unrealistic situation in economic behaviour).

Mathematically our results extend a classical theorem of Frobenius (see [2, Chapter VI]). More specifically, we generalize the work of Nikliborc [5], Thomas [8] and Tsuji [9] by using the approach to generalized differentiation as found in Serrin [7]. Our methods also extend to spaces of infinite dimensions in which case we can obtain results useful in the study of the time evolution of economic systems. An (alternative) axiomatic approach to utility theory has been studied in recent years by von Neumann and Morgenstern [10], Herstein and Milnor [3], and others. It is a pleasure to thank Professor L. Hurwicz for suggesting this problem and for invaluable help with its study. This research was partially supported by the grants NSF GP 3904 and AFOSR 883-65.

1. Statement of the problem for finite dimensional commodity spaces. Let $x=\left(x_{1}, \cdots, x_{n}\right)$ and $p=\left(p_{1}, \cdots, p_{n}\right)$ be vectors in $\bar{R}^{n}$ and $R^{n}$ respectively with real nonnegative entries. Denote by $\left(p, p_{n+1}\right)$ and $(x, y)$ vectors in $R^{n} \times R^{1}$ and $\bar{R}^{n} \times \bar{R}^{1}$. Then we make following usual definitions of mathematical economics:

(a) A demand function $\bar{f}(p, m)$ is a mapping of $R^{n} \times R^{1} \rightarrow \bar{R}^{n} \times \bar{R}^{1}$ which satisfies the identity:

$$
(p, 1) \cdot \bar{f}(p, m)=m \quad \text { for all }(p, m) \in R^{n} \times R^{1}
$$

where the dot denotes the usual scalar multiplication in $(n+1)$ dimensions. Economically this identity means: (i) an $(n+1)$ st com- 
modity, viz. money, is chosen as a standard and the prices of other commodities are expressed relative to this standard, (ii) if we set $f(p, m)=\left(f_{1}, \cdots, f_{n}\right)$, then $\bar{f}(p, m)$ is uniquely determined by $f(p, m)$.

(b) For fixed $(p, m)$, the budget set

$$
B_{p, m}=\left\{(x, y) \mid(x, y) \in \bar{R}^{n} \times \bar{R}^{1}, \quad(p, 1)(x, y) \leqq m\right\} .
$$

(c) A utility function $U(x, y)$ is a real-valued continuous function defined on $\bar{R}^{n} \times \bar{R}^{1}$ such that for each $(p, m) \max _{B_{p, m}} U(x, y)$ is attained at $(x, y)=\bar{f}(p, m)$.

It is the object of this work to determine conditions on $f(p, m)$, (assumed given), that guarantee the existence of such a utility function. We proceed in the following way: In order to solve an initial value problem for a given system of nonlinear partial differential equations, we find a unique solution for an associated integral equation. Under suitable conditions, this solution is shown to satisfy the given differential equations. Here we require a result on the validity of the chain rule for generalized derivatives. The utility function is then constructed following a procedure of Hurwicz and Uzawa [4]. This construction is then extended in a straightforward manner to spaces of infinite dimension.

2. On the validity of the chain rule for generalized differentiation. $p=\left(p_{1}, \cdots, p_{n}\right)$ stands for a point in $R^{n}$, while $m$ stands for a real number; $(p, m)$ is a point in $R^{n+1}$. We define the two projection maps $\pi_{p}, \pi_{m}$ by

$$
\pi_{p}[(p, m)]=p, \quad \pi_{m}[(p, m)]=m .
$$

Definition. Let $M(p)$ be a locally summable function defined almost everywhere on $R^{n}$. We say that $M(p)$ is C.L. if the distribution derivatives of $M(p)$ are measures and $M(p)$ is equal almost everywhere to a function $M(p)$, where, for each index $i, M(p)$ is continuous in $p_{i}$ for almost all values of the remaining variables.

We further say that $M(p)$ is A.C.L. if $M(p)$ is C.L. and its distribution derivatives are functions. In both cases we denote the absolutely continuous part of the derivative vector-valued measure by $M_{, p}(p)$.

A function is A.C.L. if and only if it is strongly differentiable and obviously every Lipshitz function is A.C.L. If we call a function whose distribution derivatives are measures, weakly differentiable, then the class of functions which are C.L. is a class lying between the weakly and strongly differentiable functions. These functions have been studied extensively by Goffman (see [1]). 
Theorem 1. If $\Omega$ is a measurable subset of $R^{n}, M(p)$ is C.L. and measure $M(\Omega)=0$, then $M_{, p}(p)=0$ a.e. on $\Omega$.

The above theorem generalizes a known result for the case when $M(p)$ is A.C.L. and $M(p)$ is constant on $\Omega$.

THEOREM 2. If

(i) $f(p, m)$ satisfies a Lipshitz condition on $R^{n+1}$,

(ii) There exist Borel sets $\odot$ and $\mathfrak{T}$ contained in $R^{n+1}$ such that

$$
\begin{aligned}
& n \text {-meas. } \pi_{p}(\odot)=1 \text {-meas. } \pi_{m}(\mathfrak{T})=0, \\
& \frac{\partial f}{\partial p}(p, m) \text { exists except on } \odot, \\
& d f(p, m) \text { exists except on } \odot \cup \mathfrak{T},
\end{aligned}
$$

(iii) $M(p)$ is $\{$ C.L./A.C.L., then $f(p, M(p))$ is $\{$ C.L./A.C.L. $\}$ and

$$
f(p, M(p))_{, p}=\frac{\partial f}{\partial p}(p, M(p))+\frac{\partial f}{\partial m}(p, M(p)) M_{, p}(p) \text { a.e. }
$$

where we take

$$
\frac{\partial f}{\partial t}(p, M(p)) M_{, p}(p)=0 \quad \text { wherever } M_{, p}(p)=0
$$

Note that condition (ii) is fulfilled if $f$ is a Lipshitz function which is independent of $p$.

The above theorem can easily be generalized to the case where $M(p)$ assumes values in $R^{m}$.

3. The system of differential equations. We consider the system of partial differential equations

$$
\operatorname{grad} M(p)=f(p, M)
$$

with initial condition $M\left(p_{0}\right)=M_{0}$ and we look for a solution on the portion of $R^{n}$ where $p \geqq 0$. The following conditions on $f(p, m)$ are relevant:

(A) The conditions (i) and (ii) of Theorem 2.

(B) $S_{i j}=S_{j i}$ except on $\odot \cup \mathfrak{T}$, where

$$
S_{i j}=\frac{\partial f_{i}}{\partial p_{j}}+\frac{\partial f_{i}}{\partial m} f_{j}
$$

(B') $S_{i j}=S_{j i}$ except on $\odot$, where we have set 


$$
\frac{\partial f}{\partial m}=0 \quad \text { on } \mathfrak{T}
$$

Theorem 3. If (A) and (B) hold and in addition $f(p, m) \neq 0$ except on $\odot$ then (1) has a unique continuously differentiable solution.

If (A) and ( $\left.\mathrm{B}^{\prime}\right)$ hold then (1) has a unique continuously differentiable solution.

4. Construction of a utility function. Before proceeding to a statement of the existence theorem, we consider the following additional hypothesis on $f(p, m)$ :

(C) The matrix $\left(S_{i j}\right)$ is negative semidefinite except on pUTr.

THEOREM 4. If $f(p, m)$ satisfies (C) together with one of the two sets of conditions given in Theorem 3 then a utility function, $U(x, y)$, defined on the range of $\bar{f}(p, m)$ exists.

To construct such a utility function we modify a procedure of Hurwicz and Uzawa [4]. Consider the system (3) and its unique solution with the initial condition $M\left(p_{0}\right)=M_{0}$, which we denote $M\left(p ; p_{0}, M_{0}\right)$. Then for fixed $p_{0} \in R^{n}$, we define the utility function for each $(x, y)$ in the range of $\bar{f}(p, m)$ as follows

$$
U_{p_{0}}(x, y)=M\left(p_{0}: \bar{f}^{-1}(x, y)\right) .
$$

Here $\bar{f}^{-1}(x, y)$ denotes any point $\left(p^{\prime}, m^{\prime}\right)$ such that $\bar{f}\left(p^{\prime}, m^{\prime}\right)=(x, y)$. The uniqueness of the solution of (3) guarantees the existence of a point $M_{0}$ such that $M\left(p_{0} ; \bar{f}^{-1}(x, y)\right)=M\left(p_{0} ; p_{0}, M_{0}\right)$.

5. Utility functions in an infinite dimensional commodity space. Let $\chi$ be a reflexive Banach space over the reals with conjugate space denoted $\chi^{*}$. Let $B$ be a continuous bounded possibly nonlinear mapping from $\chi_{+} \rightarrow \chi_{+}^{*}$ such that $(x, B x)>0(x \neq 0)$, where $\chi_{+}$and $\chi_{+}^{*}$ are positive cones.

Let the budget set $B_{x}=\left\{x^{*} \mid\left(x, x^{*}\right) \leqq(x, B x)\right\}$ where $\left(x, x^{*}\right)$ denotes the inner product of $x \in \chi_{+}$and $x^{*} \in \chi_{+}^{*}$.

Definition. A utility functional $U\left(x^{*}\right)$ is a real-valued continuous mapping $X_{+}^{*} \rightarrow R^{1}$ such that for each $x \in \chi_{+}, \max _{B_{x}} U\left(x^{*}\right)$ is attained at $B x$.

We now determine abstract conditions on $B$ that guarantee the existence of such a functional.

Definition. An operator $B u$ is derivable from an indirect utility functional $\phi(x)$ if the Gateaux derivative of $\phi(x)$ in the direction $v$ is $(v, B x)$ for every $v \in \chi$. 
Lemma 3 (An InTEgrability Condition). $B$ is derivable from the indirect utility function $\phi(x)$ if and only if the following identity holds for each $x, y \in \chi$

$$
\int_{0}^{1}(x B(s x)) d s-\int_{0}^{1}(y, B(s y)) d s=\int_{0}^{1}(x-y, B(y+s(x-y))) d s .
$$

Furthermore we can set $\phi(x)=\int_{0}^{1}(x, B(s x)) d s$.

We now impose the following condition on $B$ :

(II) Monotonicity $(u-v, B u-B v) \leqq 0$, with equality only if $u=v$,

(III) If $u_{n} \rightarrow u$ weakly in $\chi$ and $B u_{n} \rightarrow B u$ strongly in $\chi^{0}$, then $u_{n} \rightarrow u$ strongly.

(IV) $B^{-1}$ is a bounded operator, (where defined).

THEOREM 4. Let the operator $B$ satisfy the conditions I, II, III, IV, then a continuous utility functional $U\left(x^{*}\right)$, defined on the range of $B$, exists.

Indeed we may define

$$
U\left(x^{*}\right)=k_{0}-\int_{0}^{1}(x, B(s x)) d s
$$

where $x^{*}=B x$, and $k_{0}$ is a positive constant.

\section{BIBLIOGRAPHY}

1. C. Goffman, Non parametric surfaces given by linearly continuous functions, Acta Math. 103 (1960), 271-291.

2. P. Hartman, Ordinary differential equations, Wiley, New York, 1964.

3. I. Herstein and J. Milnor, An axiomatic approach to measurable utility, Econometrica 17, (1953), 291-296.

4. L. Hurwicz and H. Uzawa, The integrability conditions for demand functions, (to appear). 41-49.

5. W. Nikliborc, Sur les équations aux differentiables totales, Studia Math. 1 (1929),

6. P. Samuelson, The problem of integrability in utility theory, Economica 17 (1950), 355-385.

7. J. Serrin, Theory of differentiation, (Mimeographed Lecture Notes), University of Minnesota, Minneapolis, Minn.

8. T. Y. Thomas, Systems of total differential equations defined over simply connected domains, Ann. of Math. 35 (1934) 730-734.

9. M. Tsuji, On a system of total differential equations, Japan. J. Math., 19 (1948), 383-393.

10. J. von Neumann and O. Morgenstern, Theory of games and economic behavior, Princeton Univ. Press, Princeton, N. J., 1944.

University of Minnesota 